\title{
Research on research funding: An imperative for science and society
}

$$
\text { Anna Severin }{ }^{1,2,3} \text {, Matthias Egger }{ }^{1,4,5}
$$

${ }^{1}$ Institute of Social \& Preventive Medicine, University of Bern, Bern, Switzerland

${ }^{2}$ Strategy Support, Swiss National Science Foundation, Bern, Switzerland

${ }^{3}$ Department of Science, Technology, Engineering and Public Policy, University College London, London, UK

${ }^{4}$ Research Council, Swiss National Science Foundation, Bern, Switzerland

${ }^{5}$ Population Health Sciences, Bristol Medical School, University of Bristol, Bristol, UK

\section{CORRESPONDING AUTHOR}

Anna Severin

Swiss National Science Foundation

Wildhainweg 3

3012 Bern, Switzerland

anna.severin@snf.ch

\section{WORD COUNT}

Text 901 words, 8 references, 1 figure

\section{COMPETING INTERESTS}

The authors declare no competing interests.

\begin{abstract}
AUTOR CONTRIBUTIONS
AS and ME conceived the idea for this editorial. AS wrote the first draft. ME contributed to additional content creation and to the editing of the final draft.
\end{abstract}

\section{ACKNOWLEDGEMENTS}

The authors declare no acknowledgements.

\section{FUNDING}

No research funding was obtained to undertake this editorial.

\section{ETHICAL APPROVAL INFORMATION}

Not applicable.

\section{DATA SHARING STATEMENT}

Not applicable. 
Research in medicine and public health is essential for the future wellbeing of society. In sports medicine, research has, for example, shown that psychological, social and contextual factors all influence recovery processes after sport-related injuries. ${ }^{1}$ Such an understanding is central to optimise rehabilitation and improve outcomes and quality of life.

Many members of the BJSM community are well versed in the research funding world. In research funding, peer review of grant applications is considered the best practice for deciding which projects or scholars are funded. There are, however, concerns about the validity of peer review and research evaluation. A recent systematic review indicated that grant peer review in the health sciences suffers from biases, conservatism and is a weak predictor of future research performance. ${ }^{2}$ We acknowledge that maximising effectiveness and fairness of public research funding is challenging and in this editorial, we argue that research on research funding is required to reduce biases and conservatism and increase the efficiency of grant review.

\section{Does peer review of grant proposals work?}

There are inherent weaknesses in grant review, which raise the question if it is the best method for allocating research funding. As funding organisations only recently have begun to investigate their review processes, most evidence on these challenges is anecdotal. Additionally, not much is known about how challenges can be addressed to improve evaluation and decision-making.

Biases in grant review refer to the potential influence of factors on funding decisions that are unrelated to the actual quality of grant applications. Biases can be conscious or unconscious and stem from the applicant's or the reviewer's characteristics, including their institutional affiliation, research field, nationality, gender, age, or ethnicity. Potential discrimination against female scientists is the most frequently studied bias. The Swiss National Science Foundation (SNSF) recently found that male applicants received more favourable scores from peer reviewers than female applicants. ${ }^{3}$ Male reviewers awarded higher scores than female reviewers, particularly for male applicants (Figure 1). The Swiss data support the 'gender matching hypothesis', where male peer reviewers give higher scores to male researchers, and female reviewers do the same for female applicants. However, the data are observational, and other explanations than bias against women exist. Indeed, differences were attenuated after adjusting for potential confounders. ${ }^{3}$ 


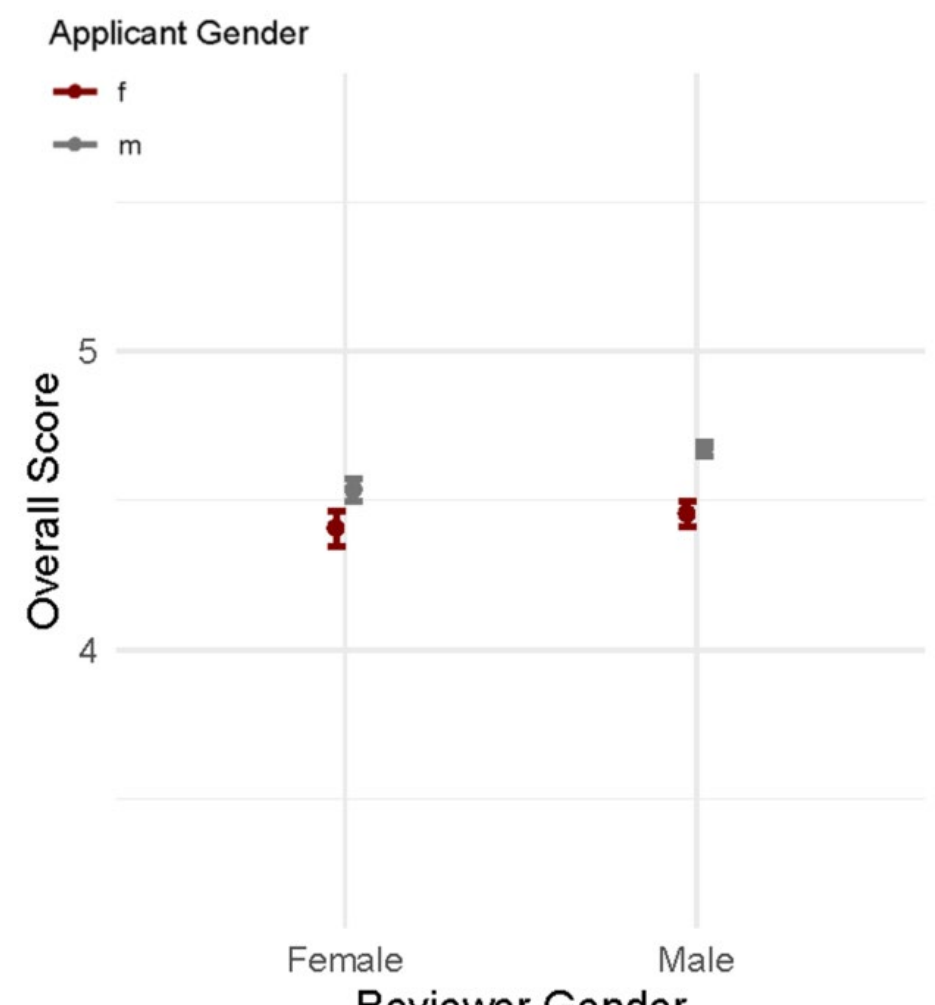

Figure 1. Gender differences in external peer review scores by gender of the main applicant and gender of the expert reviewer. Scores range from 1 (worst) to 6 (best). Mean overall scores with 95\% confidence intervals are shown.

Data from 38250 external peer review reports used for project funding at the Swiss National Science Foundation, 2006-2016. Adapted from Severin et al. ${ }^{3}$

The limited efficiency of grant review in selecting the best research or people is another challenge. A study from the Finnish Research Council for Health found that the discussion of proposals by evaluation panels, which are expensive and time-consuming, did not improve the reliability of the evaluation compared to merely using the mean score of external reviewers. ${ }^{4}$

Conservatism relates to grant review putting innovative and risky grant proposals at a disadvantage. While most evidence on conservatism is anecdotal, interviews with panelists at the European Research Council revealed that, because funding budgets are limited, reviewers tend to select proposals where the research is more likely to succeed, rather than rewarding ambitious, innovative ideas. ${ }^{5}$ This is also referred to as incrementalism. At the National Institutes of Health $(\mathrm{NIH})$, there is concern about the falling numbers of innovative and risky grant proposals. Indeed, competitive pressures and low success rates may lead to conservative applications that focus on short-term success. ${ }^{6}$

\section{A call for research on research funding}

The body of knowledge on peer review comes from studies that cannot control for the difficult to measure factors that influence funding decisions, including the reputation of the applicants, or 
their field of research. We call on funding agencies to invest in innovative experimental and observational studies that may allow causal inferences and can inform policy to reduce bias and conservatism and increase efficiency. Such studies may include randomised or quasi-randomised experiments, simulations, and approaches such as inverse probability weighting in longitudinal studies, for example of cohort studies of the impact of career funding among scholars, regression-discontinuity designs, or matching, for example of funded and rejected proposals close to the funding line.

Similar to clinical research, funders could randomise grant proposals to treatment groups with experimental review and a control group following the standard approach. For example, the effectiveness of blinding reviewers to the identity of applicants and their affiliations in reducing biases related to reputation, research field, nationality, gender, age, or ethnicity could be examined. Such randomised studies have been done for journal submissions ${ }^{7}$, but not for grant proposals.

A recent collaborative study by researchers and the Canadian Institutes of Health Research (CIHR) produced quasi-experimental and hence robust evidence that gender gaps in grant funding at the CIHR were attributable to the less favourable assessment of track records of women, and not of the quality of their proposed research. ${ }^{8}$ Other research should, for example, examine how the bias against interdisciplinary or risky proposals can be reduced, the role of lottery approaches to selecting proposal for funding, or the potential of training panel members or peer reviewers.

It is time for research funders to tackle the limitations inherent in peer review and grant evaluation that prevent promising research from being funded. This will take dedicated funding. If there are national research funding panels for cancer research, genetics research and physical activity research, why are there no panels for research to make grant funding more equitable and effective? Funders - for sports medicine grants and beyond - must be creative when investigating the merit of different evaluation strategies. We call for research and for letting those research findings guide funding reforms to promote grant review that supports the best research. 


\section{References}

1 Truong LK, Mosewich AD, Holt CJ, Le CY, Miciak M, Whittaker JL. Psychological, social and contextual factors across recovery stages following a sport-related knee injury: a scoping review. Br J Sports Med 2020; published online Feb 14. DOI:10.1136/bjsports-2019-101206.

2 Guthrie S, Ghiga I, Wooding S. What do we know about grant peer review in the health sciences? F1000Research 2017; 6: 1335.

3 Severin A, Martins J, Heyard R, Delavy F, Jorstad A, Egger M. Gender and other potential biases in peer review: cross-sectional analysis of 38250 external peer review reports. $B M J$ Open 2020; 10: e035058.

4Fogelholm M, Leppinen S, Auvinen A, Raitanen J, Nuutinen A, Väänänen K. Panel discussion does not improve reliability of peer review for medical research grant proposals. Journal of Clinical Epidemiology 2012; 65: 47-52.

5 Luukkonen T. Conservatism and risk-taking in peer review: Emerging ERC practices. Research Evaluation 2012; 21: 48-60.

6Scarpa T. Research funding: peer review at NIH. Science 2006; 311: 41.

7 Tomkins A, Zhang M, Heavlin WD. Reviewer bias in single- versus double-blind peer review. Proc Natl Acad Sci USA 2017; 114: 12708-13.

8 Witteman HO, Hendricks M, Straus S, Tannenbaum C. Are gender gaps due to evaluations of the applicant or the science? A natural experiment at a national funding agency. The Lancet 2019; 393: 531-40. 